\title{
Determination of Surface Potential and Electrical Double-Layer Structure at the Aqueous Electrolyte-Nanoparticle Interface
}

\author{
Matthew A. Brown, ${ }^{1, *}$ Zareen Abbas, ${ }^{2}$ Armin Kleibert, ${ }^{3}$ Richard G. Green, ${ }^{4}$ Alok Goel,,${ }^{1}$ Sylvio May, ${ }^{5}$ and Todd M. Squires ${ }^{6}$ \\ ${ }^{1}$ Laboratory for Surface Science and Technology, Department of Materials, \\ ETH Zürich, Vladimir-Prelog-Weg 5, CH-8093 Zurich, Switzerland \\ ${ }^{2}$ Department of Chemistry and Molecular Biology, University of Gothenburg, \\ SE-41296 Gothenburg, Sweden \\ ${ }^{3}$ Swiss Light Source, Paul Scherrer Institut, CH-5232 Villigen PSI, Switzerland \\ ${ }^{4}$ Measurement Science and Standards, National Research Council Canada, Ottawa, \\ Ontario K1A 0R6, Canada \\ ${ }^{5}$ Department of Physics, North Dakota State University, Fargo, North Dakota 58108-6050, USA \\ ${ }^{6}$ Chemical Engineering Department, University of California Santa Barbara, Santa Barbara, \\ California 93106-5080, USA
}

(Received 31 July 2015; revised manuscript received 4 December 2015; published 28 January 2016)

\begin{abstract}
The structure of the electrical double layer has been debated for well over a century, since it mediates colloidal interactions, regulates surface structure, controls reactivity, sets capacitance, and represents the central element of electrochemical supercapacitors. The surface potential of such surfaces generally exceeds the electrokinetic potential, often substantially. Traditionally, a Stern layer of nonspecifically adsorbed ions has been invoked to rationalize the difference between these two potentials; however, the inability to directly measure the surface potential of dispersed systems has rendered quantitative measurements of the Stern layer potential, and other quantities associated with the outer Helmholtz plane, impossible. Here, we use x-ray photoelectron spectroscopy from a liquid microjet to measure the absolute surface potentials of silica nanoparticles dispersed in aqueous electrolytes. We quantitatively determine the impact of specific cations $\left(\mathrm{Li}^{+}, \mathrm{Na}^{+}, \mathrm{K}^{+}\right.$, and $\left.\mathrm{Cs}^{+}\right)$in chloride electrolytes on the surface potential, the location of the shear plane, and the capacitance of the Stern layer. We find that the magnitude of the surface potential increases linearly with the hydrated-cation radius. Interpreting our data using the simplest assumptions and most straightforward understanding of Gouy-Chapman-Stern theory reveals a Stern layer whose thickness corresponds to a single layer of water molecules hydrating the silica surface, plus the radius of the hydrated cation. These results subject electrical double-layer theories to direct and falsifiable tests to reveal a physically intuitive and quantitatively verified picture of the Stern layer that is consistent across multiple electrolytes and solution conditions.
\end{abstract}

DOI: 10.1103/PhysRevX.6.011007

\section{INTRODUCTION}

The formation of excess positive or negative charge on solid surfaces upon contact with aqueous solutions gives rise to an electrical double layer (EDL) as ions in the adjacent electrolyte rearrange to screen the charge. The microscopic structure of this EDL is difficult to interrogate experimentally [1-5] and is extensively debated in the literature because of its fundamental and technological significance in controlling surface structure [6,7], regulating interfacial reactivity [8] and colloid-colloid interactions [9], governing transport in microfluidics [10] and

*matthew.brown@mat.ethz.ch

Published by the American Physical Society under the terms of the Creative Commons Attribution 3.0 License. Further distribution of this work must maintain attribution to the author(s) and the published article's title, journal citation, and DOI.
Subject Areas: Materials Science, Physical Chemistry, Soft Matter

nanofluidics [11], and mitigating drug-carrier cell interactions [12]. The EDL is also at the heart of many processes of global interest [13], including capacitive deionization of ground water $[14,15]$ and the harnessing of intermittent power sources (e.g., wind and solar) through large-scale electric double-layer capacitors [16].

An important property of the EDL that remains poorly understood is the surface potential $\left(\Phi_{0}\right)$. In dispersed nanoparticle (NP) systems, surface potentials are traditionally inferred from electrokinetic (zeta) potentials, which depend only on the charge in the diffuse part of the EDL [17], since the direct experimental determination of surface potentials is widely believed to be impossible [17-19]. In the absence of detailed experimental results, the burden of determining surface potentials for these systems falls to theory, which often depends on parameters that cannot be measured directly, but are instead chosen to fit measured data - a less than ideal situation. Given the importance of 
the surface potential (relative to the bulk electrolyte) for suspension stability, adsorption kinetics, electrochemistry, heterogeneous catalysis, ion exchange, electric doublelayer capacitors, and other surface-mediated processes including charge transfer, direct measurements of the surface potential of nanoparticles - and its dependence on, e.g., specific ions and $p \mathrm{H}-$ could enable a much deeper and more rational approach to the understanding and design of these systems.

$\mathrm{X}$-ray photoelectron spectroscopy (XPS) is a powerful analytical technique that uses a monochromatic incident $\mathrm{x}$ ray to ionize the sample, causing photoelectrons to be emitted with energies that depend upon their binding energy (BE) within the sample's specific orbitals. Because XPS is traditionally performed in ultrahigh vacuum, measurements with liquid samples have been limited to a few instruments worldwide [20-24]. Recently, however, we demonstrated that aqueous suspensions of nanoparticles (colloids) can be passed within a liquid microjet through the x-ray beam, enabling the unique analytical capabilities of XPS to be extended to aqueous NP dispersions [25]. With this setup, we have published proof-ofprinciple measurements demonstrating the unique ability of XPS to access relative surface potentials of dispersed NPs [25]. Here, we build on these results using XPS to make the unprecedented measurement of the absolute surface potentials of silica $\left(\mathrm{SiO}_{2}\right)$ NPs in different alkali chloride electrolytes (specific ion effects) by measuring the surface potential under conditions where the NPs are uncharged, for which surface potentials should be zero.

Our measurements reveal a pronounced ion specificity in the nanoparticles' surface potentials under otherwise identical conditions. Differences correlate directly with the hydrated radii of each specific cation, which naturally suggests a simple and intuitive mechanism: The hydratedcation radius sets a distance of closest approach to the NP surface, which is generally identified as the thickness of the Stern layer. These ion-specific effects suggest the presence of forces mediated by the structure of the solvent between pairs of counterions and between counterions and the surface. To account for these so-called hydration forces, we use a modified Poisson-Boltzmann (PB) model that adds a nonelectrostatic, Yukawa-like, ion-ion interaction to the Coulomb pair potential. In excellent agreement with our experiments, the modified PB model reproduces the cationspecific surface potentials and Stern layer thicknesses.

\section{EXPERIMENTAL SETUP}

A complete description of the experimental procedures is given in Appendix A1-A4. Here, only the aspects unique to the present experiments (measure of $\Phi_{0}$ by XPS) are discussed.

XPS measurements are performed at the SIM beam line [26] of the Swiss Light Source using a near ambient pressure photoemission end station [20]. Ionization of the Si $2 p$ and $\mathrm{O} 1 s$ orbitals is done simultaneously using a combination of primary $(420 \mathrm{eV}$ for $\mathrm{Si} 2 p)$ and secondary $(840 \mathrm{eV}$ for $\mathrm{O} 1 s)$ radiation. This approach results in the Si $2 p$ and $\mathrm{O} 1 s$ photoelectrons having virtually the same photoelectron kinetic energy, and thereby ensures the NPs and aqueous electrolytes are sampled from the same probe depth into solution. The $\mathrm{Si} 2 p$ photoelectrons originate exclusively from the NPs (see Appendix A 4), whereas the $\mathrm{O} 1 s$ component of the NPs cannot be resolved from that of the electrolyte [27]: $\mathrm{O} 1 \mathrm{~s}$ photoelectrons in the present experiments are therefore assumed to originate predominantly from water. Both the Si $2 p$ and O $1 s$ photoelectrons experience an identical potential change when escaping the liquid sample into vacuum (the unknown and highly debated surface potential of water). The Si $2 p$ photoelectrons shift in energy with the nanoparticles' surface potential (potential of the NP-electrolyte interface), but the $\mathrm{O} 1 s$ does not experience this potential. When the two binding energies are measured for two different NP suspensions (wherein the NP surface potentials differ by an unknown amount $\Delta \Phi_{0}$ ), the relative energy of the Si $2 p$ orbital will be directly proportional to $\Delta \Phi_{0}$.

The fact that both the Si $2 p$ and $\mathrm{O} 1 s$ photoelectrons experience the same electrostatic potential history everywhere from the liquid microjet to the photoelectron detector, whereas the Si $2 p$ photoelectrons experience an additional potential (acceleration or deceleration) due to $\Delta \Phi_{0}$ of the NPs, makes this experimental approach insensitive to the vacuum-electrolyte surface potential and any streaming potentials established by the flowing microjet.

\section{RESULTS AND DISCUSSION}

\section{A. XPS as a quantitative analytical probe sensitive to surface potential}

In vacuum, the photoelectrons kicked out of core levels (CL) from a given sample have a known set of binding energies $\left(\mathrm{BE}_{\mathrm{CL}}^{\mathrm{sample}}\right)[28,29]$. The energies of the photoelectrons can, however, be varied by applying an external bias that effectively shifts all energy levels up (with negative applied potential) or down (positive) by creating a (surface) potential relative to the reference state of the grounded analyzer $[30,31]$. The shift follows precisely the applied potential: $+1.0(-1.0) \mathrm{V}$ of applied potential results in a $+1.0(-1.0) \mathrm{eV}$ shift in BE. The origin of this shift is well understood, and results from the (de)acceleration of the outgoing photoelectron by the electric field established by the external potential at the sample interface. A positive potential retards the outgoing photoelectron-effectively decreasing its kinetic energy (increasing the apparent BE) as it leaves the surface [Fig. 1(a)]. To demonstrate the ability of XPS to quantitatively follow this effect, we perform experiments with a $\mathrm{SiO}_{2} / \mathrm{Si}(100)$ substrate in ultrahigh vacuum. The sample consists of the native $\mathrm{SiO}_{2}$ oxide layer on a $\mathrm{Si}(100)$ substrate. Positive and 

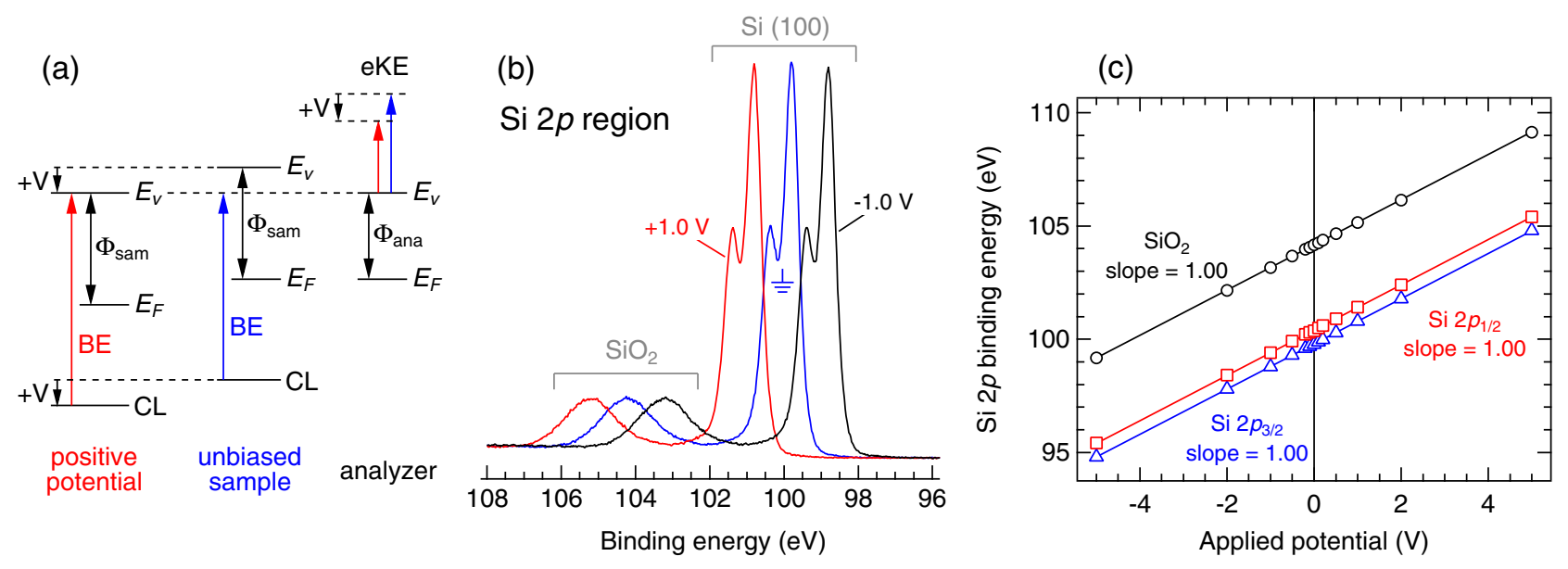

FIG. 1. Surface potential measurements by XPS. (a) Energy level diagram for the photoelectron process from an unbiased sample (blue) and from a sample under positive external potential (red). $E_{F}$, Fermi level; $E_{V}$, vacuum level; $\Phi$, work function; sam, sample; ana, hemispherical energy analyzer; eKE, photoelectron kinetic energy; BE, binding energy; CL, core level; V, applied potential. A positive bias shifts all of the sample levels down with respect to those of the analyzer, having the effect of decreasing the kinetic energy of the outgoing photoelectron. (b) $\mathrm{Si} 2 p$ spectra from a $\mathrm{SiO}_{2} / \mathrm{Si}$ (100) substrate. Blue, grounded; red, positive (1 V) bias; black, negative $(-1 \mathrm{~V})$ bias. (c) $\mathrm{Si} 2 p \mathrm{BE}$ as a function of applied potential.

negative biases up to $\pm 5.0 \mathrm{~V}$ are applied to the sample (grounded, $\pm 0.1, \pm 0.2, \pm 0.5, \pm 1.0, \pm 2.0$, and $\pm 5.0 \mathrm{~V}$ ) while recording the Si $2 p$ spectra [Fig. 1(b)]. The positions of the Si $2 p$ photoelectron peaks track the applied potentials precisely (slopes are 1.00 to two decimal places), confirming the capability of XPS to accurately monitor changes in surface potential [Fig. 1(c)].

In an electrolyte solution at $p \mathrm{H}$ conditions where the silica surface is charged, $\equiv \mathrm{Si}-\mathrm{OH}+\mathrm{OH}^{-} \leftrightarrow \equiv \mathrm{Si}-\mathrm{O}^{-}+\mathrm{H}_{2} \mathrm{O}$, the surface potential affects the apparent $\mathrm{BE}$ of the photoelectrons in precisely the same way as if an external potential were directly applied. The apparent BE of the $\mathrm{Si} 2 p$ orbital in a given salt $\left(\mathrm{BE}_{\mathrm{Si} 2 p}^{\mathrm{salt}}\right)$ shifts relative to an uncharged particle in the same electrolyte by an amount that is exactly equal to the surface potential [25,32], since the emitted photoelectron travels through, and is (de)accelerated by, the electric field in the EDL [Eq. (1)]:

$$
\mathrm{BE}_{\mathrm{Si} 2 p}^{\text {salt }}=\mathrm{BE}_{\mathrm{Si} 2 p}^{p \mathrm{H}_{\mathrm{PZC}}}+\Phi_{0}^{\text {salt }} e .
$$

Here, $\Phi_{0}^{\text {salt }}$ is the surface potential of the NP in that particular electrolyte, $\mathrm{BE}_{\mathrm{S} 2}^{p \mathrm{H}_{\mathrm{PZC}}}$ is the $\mathrm{Si} 2 p \mathrm{BE}$ of the uncharged NP in the same electrolyte, and $e$ is the elementary charge. The superscript $p \mathrm{H}_{\mathrm{PZC}}$ refers to the $p \mathrm{H}$ at the point of zero net charge, $\sim 2-3$ for silica [33,34]. Here, we take the apparent $\mathrm{Si} 2 p$ BE measured for silica NPs in $50-\mathrm{mM} \mathrm{NaCl}$ $\left(\mathrm{BE}_{\mathrm{Si} 2 p}^{\mathrm{NaCl}}\right)$ as reference, and measure the shift in $\mathrm{BE}\left(\Delta \mathrm{BE}_{\mathrm{Si} 2 p}\right)$ for the same NPs in different alkali chloride electrolytes relative to the $\mathrm{NaCl}$ value:

$$
\Delta \mathrm{BE}_{\mathrm{Si} 2 p}=\mathrm{BE}_{\mathrm{Si} 2 p}^{\mathrm{salt}}-\mathrm{BE}_{\mathrm{Si} 2 p}^{\mathrm{NaCl}}=\Delta \Phi_{0} e .
$$

Equation (2) reveals the unique ability of XPS to directly access specific ion effects upon $\Delta \Phi_{0}$-i.e., the change in surface potential brought about by a change in electrolyte under otherwise equivalent conditions-with no a priori knowledge of the oxide surface or EDL structure [25,32] (also see Fig. 1).

\section{B. Specific cation effects in alkali chloride electrolytes}

Our XPS measurements using a liquid microjet [Fig. 2(a)] of $5 \mathrm{wt} \%$ 9-nm colloidal silica at $p \mathrm{H} 10.0$ [Fig. 2(b)] reveal an unexpected linear dependence between the $\mathrm{BE}$ of the Si $2 p$ orbital and the radius of the hydrated cation in the electrolyte [Fig. 2(c)]. These shifts in Si $2 p$ BE reveal that the negative surface potential of the water-silica NP interface in monovalent chloride electrolytes exhibits pronounced specific ion effects, increasing in magnitude by as much as $150 \mathrm{mV}$ as the size of the hydrated cation is increased. These results are qualitatively supported by similar trends in zeta potentials as determined by us (Table I) [35] and others [36].

\section{Absolute surface potentials}

Figure 2 shows that the silica NP surface potential varies linearly with hydrated cation radius. Thus far, however, all surface potentials have been measured relative to a single (unknown) value in $\mathrm{NaCl}$. To determine the absolute

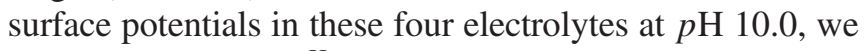
must determine $\mathrm{BE}_{\mathrm{Si} 2 p}^{p \mathrm{H}_{\mathrm{PZC}}}$ for the NP in one of the electrolytes. At $p \mathrm{H}_{\mathrm{PZC}}$, which is $\sim 2-3$ for the silicon dioxide surface [33,34], the surface potential of silica is expected to vanish $\left[\Phi_{0}^{\text {salt }}=0\right.$, Eq. (1)]. Therefore, the apparent binding energy of the $\mathrm{Si} 2 p$ orbital $\left(\mathrm{BE}_{\mathrm{Si} 2 p}^{\mathrm{salt}}\right)$ measured via XPS will reflect the true BE of the NP in aqueous solution, and thus provides the zero reference value from 

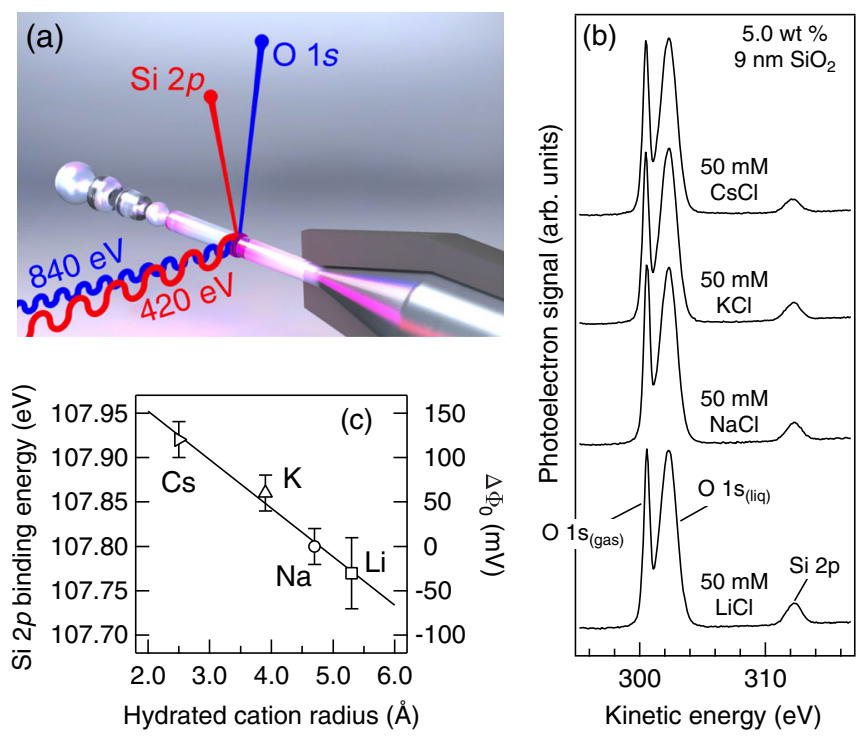

FIG. 2. XPS at the water-silica nanoparticle interface. (a) A liquid microjet delivers a stable free-flowing suspension of colloidal silica inside the measurement chamber of an XPS spectrometer. (b) $\mathrm{O} 1 s$ and $\mathrm{Si} 2 p$ spectra for $5.0 \mathrm{wt} \% \mathrm{SiO}_{2}$ in 50-mM LiCl, $\mathrm{NaCl}, \mathrm{KCl}$, and $\mathrm{CsCl}$ electrolytes at $p \mathrm{H} 10$. (c) Measured $\mathrm{Si} 2 p \mathrm{BE}$, relative to the vacuum level, as a function of hydrated cation radius [37]. The change in surface potential relative to that in $\mathrm{NaCl}$ electrolyte is shown on the righthand axis. Error bars represent the standard deviation of four repeat measurements.

which all other surface potentials can be determined absolutely. We cannot perform XPS measurements at $p \mathrm{H}_{\mathrm{PZC}}$, however, due to the limited stability of our colloidal silica over the approximately hour-long time frame of each experiment. Instead, we perform a series of experiments between $p \mathrm{H} 10.0$ and 3.5 for $5 \mathrm{wt} \% \mathrm{SiO}_{2}$ in $50-\mathrm{mM} \mathrm{NaCl}$ (open black markers of Fig. 3) and extrapolate the results to $p \mathrm{H}_{\mathrm{PZC}}=3$. Any uncertainty in the $p \mathrm{H}_{\mathrm{PZC}}$ of silica introduces only minor error, since the surface charge density (SCD) (Fig. 4) and surface potential are negligible in the $p \mathrm{H}$ window centered around 3, particularly when

Table I. TEM and small-angle x-ray scattering (SAXS) measured NP diameters, zeta potentials ( $\zeta$ pot), and surface charge densities (SCD).

\begin{tabular}{lcccc}
\hline \hline Salt & $\begin{array}{c}\text { TEM } \\
\text { diameter } \\
(\mathrm{nm})\end{array}$ & $\begin{array}{c}\text { SAXS } \\
\text { diameter }^{\mathrm{a}, \mathrm{b}} \\
(\mathrm{nm})\end{array}$ & $\begin{array}{c}\zeta \text { pot }^{\mathrm{a}, \mathrm{c}, \mathrm{d}} \\
(\mathrm{mV})\end{array}$ & $\begin{array}{c}\text { SCD } \\
\left(\sigma_{\mathrm{NP}}\right)^{\mathrm{b}, \mathrm{d}} \\
\left(\mathrm{C} / \mathrm{m}^{2}\right)\end{array}$ \\
\hline $\mathrm{LiCl}$ & $9.2(1.5)$ & $8.7(2.1)$ & $-53.8(3.3)$ & $-0.172(0.001)$ \\
$\mathrm{NaCl}$ & $9.0(1.6)$ & $9.2(1.9)$ & $-48.5(4.2)$ & $-0.173(0.003)$ \\
$\mathrm{KCl}$ & $9.0(1.4)$ & $9.1(1.8)$ & $-44.5(4.5)$ & $-0.179(0.003)$ \\
$\mathrm{CsCl}$ & $9.2(1.4)$ & $9.4(1.8)$ & $-40.0(4.1)^{\mathrm{e}}$ & $-0.188(0.001)$ \\
\hline \hline
\end{tabular}

${ }^{\mathrm{a}}$ From Ref. [35].

${ }^{\mathrm{b}} 5.0 \mathrm{wt} \%$ suspensions.

${ }^{\mathrm{c}} 2.0 \mathrm{wt} \%$ suspensions.

${ }^{\mathrm{d}}$ At $p \mathrm{H} 10.0 \pm 0.1$

${ }^{\mathrm{e}}$ At $p \mathrm{H} 9.9 \pm 0.1$.

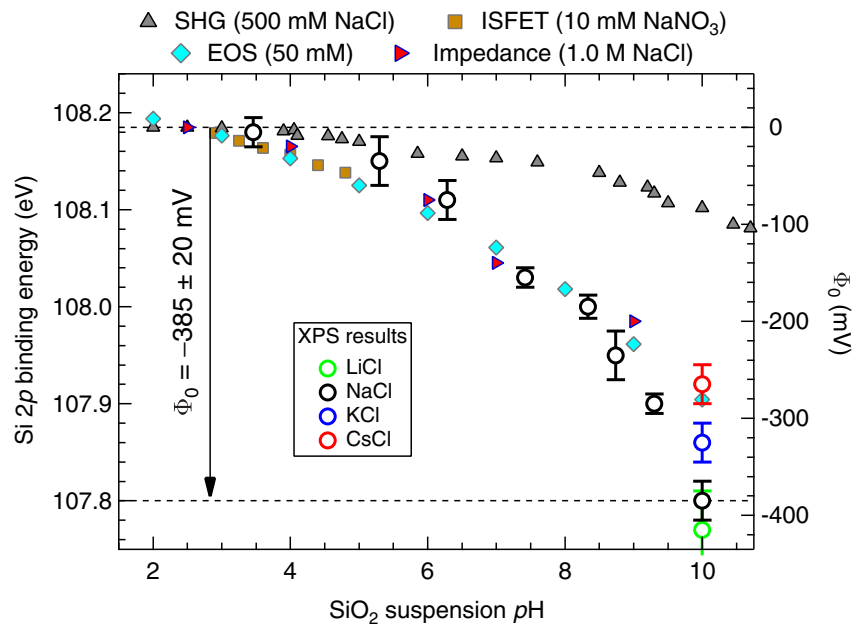

FIG. 3. $p \mathrm{H}$-dependent absolute surface potential at the silicaelectrolyte interface. Si $2 p$ binding energy (left-hand axis) and absolute surface potential (right-hand axis) at the water-silica interface for 9-nm $\mathrm{SiO}_{2}$ in 50-mM NaCl electrolyte (open black markers) as a function of bulk suspension $p \mathrm{H}$. Open markers: Surface potentials at $p \mathrm{H} 10.0$ in $50-\mathrm{mM} \mathrm{LiCl}$ (green), $\mathrm{KCl}$ (blue), and $\mathrm{CsCl}$ (red). Error bars represent the standard deviation of two measurements (four at $p \mathrm{H}$ 10.0). Solid markers: Surface potentials for various extended $\mathrm{SiO}_{2}$ surfaces as reported in the literature: SHG [38], ISFET [39], EOS [40], and Impedance [41].

compared to that at $p \mathrm{H} 10$. Our measurements reveal the absolute surface potential of $9-\mathrm{nm}^{\mathrm{SiO}}{ }_{2}$ at $p \mathrm{H} 10.0$ in $50-\mathrm{mM} \mathrm{NaCl}$ electrolyte to be $-385 \pm 20 \mathrm{mV}$. The absolute surface potentials for the other alkali chloride electrolytes follow directly from their measured $\Delta \Phi_{0}$ (Table II, open markers in Fig. 3 at $p \mathrm{H} \mathrm{10.0).}$

The overall shape of the $\Phi_{0}(p \mathrm{H})$ response measured by XPS is consistent with several earlier studies that report the measurement of surface potentials for extended silica

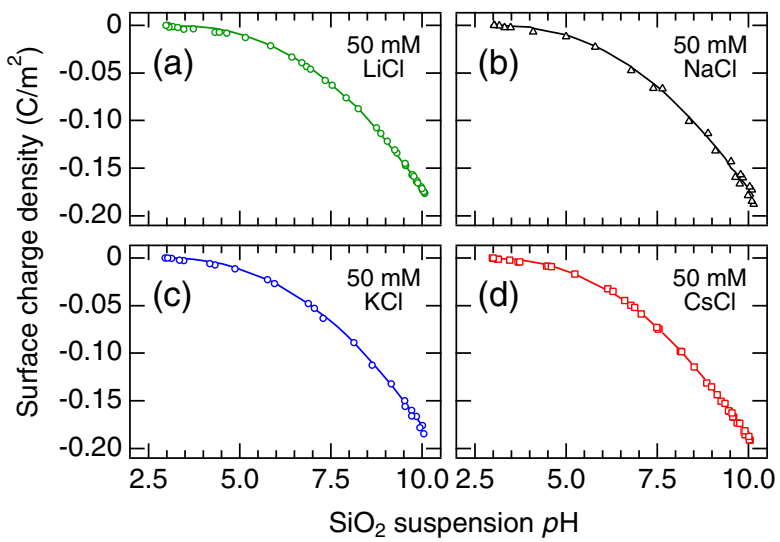

FIG. 4. $\quad p \mathrm{H}$-dependent surface charge density of colloidal silica in (a) 50-mM LiCl, (b) $50-\mathrm{mM} \mathrm{NaCl}$, (c) $50-\mathrm{mM} \mathrm{KCl}$, and (d) $50-$ $\mathrm{mM} \mathrm{CsCl}$. Markers: Experimental results from three repeat measurements. Solid lines: Fits from surface complexation modeling using the basic Stern model and $4.75 \mathrm{OH}$ sites $/ \mathrm{nm}$. The point of zero charge is set at $p \mathrm{H} 3.0$ in all electrolytes $[33,34]$. 
Table II. Hydrated cation radii, Si $2 p$ binding energies and properties of the electrical double layer.

\begin{tabular}{lccccc}
\hline \hline $\begin{array}{l}\text { Salt } \\
50 \mathrm{mM}\end{array}$ & $\begin{array}{c}r_{\text {hyd }} \\
(\AA)\end{array}\left[\begin{array}{c}\text { Si } 2 p \\
\mathrm{BE}(\mathrm{eV})\end{array}\right.$ & $\begin{array}{c}\Phi_{0} \\
(\mathrm{mV})\end{array}$ & $\begin{array}{c}d_{\text {Stern }} \\
(\AA)\end{array}$ & $\begin{array}{c}C_{\text {Stern }} \\
\left(\mathrm{F} / \mathrm{m}^{2}\right)\end{array}$ \\
\hline $\mathrm{LiCl}$ & 5.3 & 107.77 & $-415(40)$ & $8.0(0.9)$ & $0.48(0.08)$ \\
$\mathrm{NaCl}$ & 4.7 & 107.80 & $-385(20)$ & $7.4(0.4)$ & $0.51(0.05)$ \\
$\mathrm{KCl}$ & 3.9 & 107.86 & $-325(20)$ & $6.0(0.4)$ & $0.63(0.05)$ \\
$\mathrm{CsCl}$ & 2.5 & 107.92 & $-265(20)$ & $4.6(0.4)$ & $0.83(0.05)$ \\
\hline \hline
\end{tabular}

surfaces (i.e., nondispersed systems) by means of lateral current flow [ion-select field-effect transistors (ISFET)] $[39,42]$, flatband voltage shifts for an electrolyte-oxidesemiconductor (EOS) system [40], and impedance methods [41] (see solid markers of Fig. 3). However, surface potentials obtained by means of the $\chi^{(3)}$ nonlinear second harmonic generation (SHG) method deviate substantially from all others (gray solid markers of Fig. 3) [38], including ours-suggesting that the $\chi^{(3)}$ SHG method is likely a measure of the electrokinetic (zeta) potential, as recently proposed by Haber and co-workers [43], and not the commonly inferred surface potential.

\section{Electrical double-layer structure}

In the Gouy-Chapman-Stern model of the EDL [17] [Figs. 5(a) and 5(b)], the surface potential of a NP (or any extended surface) is given by the sum of the potential drops across the Stern layer [bounded by the outer Helmholtz plane (OHP)] and the diffuse layer $\Phi_{\mathrm{DL}}$. The diffuse layer potential is generally determined by electrokinetic measurements [the zeta potential $(\zeta)][17,44]$ :

$$
\Phi_{0}=\Phi_{\text {Stern }}^{\text {drop }}+\zeta
$$

Since no ions are contained within the Stern layer [17], we assume it acts as a parallel-plate capacitor,

$$
\Phi_{\text {Stern }}^{\mathrm{drop}}=\sigma_{\mathrm{NP}}\left(d_{\mathrm{Stern}} / \varepsilon \varepsilon_{0}\right),
$$

where $d_{\text {Stern }}$ is the thickness of the Stern layer (the distance between the OHP and the NP surface), and $\varepsilon$ and $\varepsilon_{0}$ are the dielectric constant of water at the silica interface (43.0) [45] and the vacuum permittivity $\left(8.85419 \times 10^{-12} \mathrm{~F} / \mathrm{m}\right)$, respectively. Combining Eqs. (3) and (4) gives the thickness of the Stern layer:

$$
d_{\mathrm{Stern}}^{\text {salt }}=\left(\Phi_{0}^{\text {salt }}-\zeta^{\text {salt }}\right)\left(\varepsilon \varepsilon_{0} / \sigma_{\mathrm{NP}}^{\text {salt }}\right) .
$$

Taking the XPS-determined surface potentials at $p \mathrm{H} 10.0$ (Table II) and the $\zeta^{\text {salt }}$ and $\sigma_{\mathrm{NP}}^{\text {salt }}$ of Table I reveals $d_{\text {Stern }}$ to increase linearly with the hydrated cation radius [Fig. 5(c), Table III. In all cases, the distance between the OHP and the NP surface exceeds one hydrated cation radius by $2.1-2.7 \AA$ (the thickness of approximately one molecular layer of water

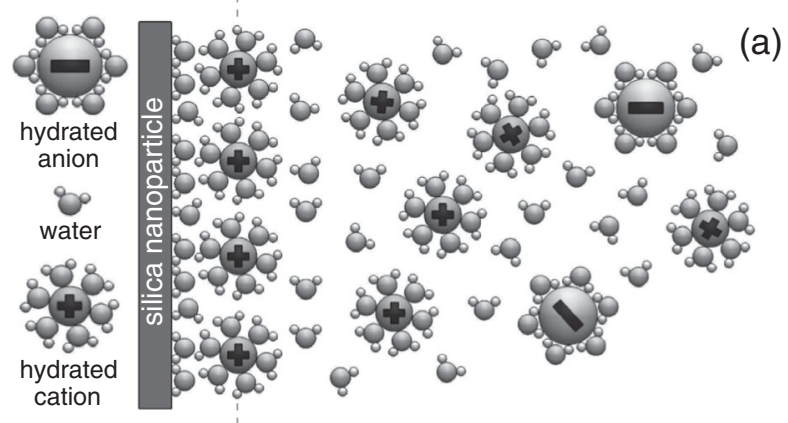

(a)

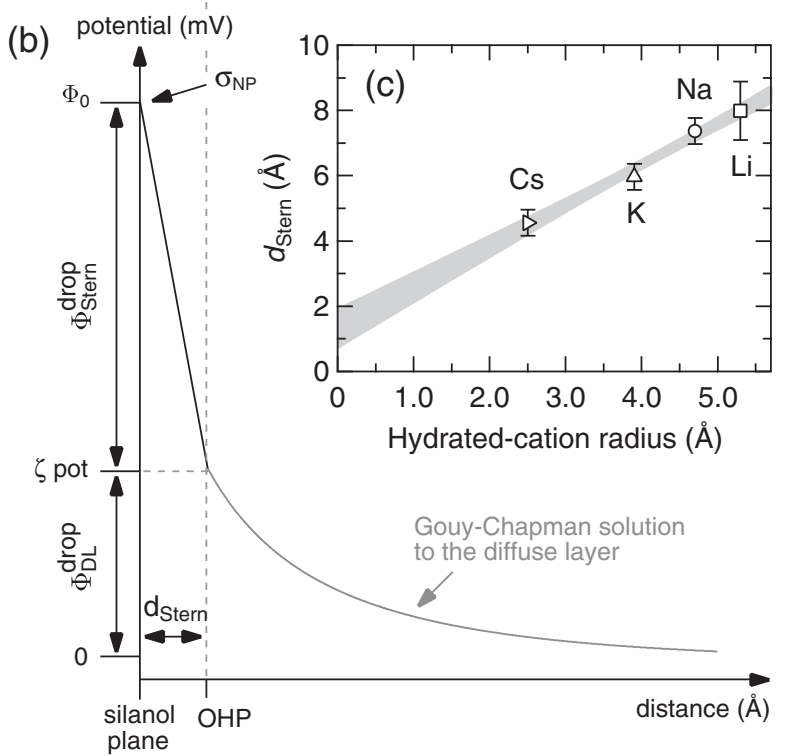

FIG. 5. Gouy-Chapman-Stern model of the electrical double layer. (a) The structure of the electrical double layer takes the form of a charged silanol plane and an outer Helmholtz plane (OHP), which represents the distance of closest approach of ions [17]. A diffuse layer of hydrated ions sits outside the OHP, screening the net surface charge over a characteristic Debye length. (b) The surface potential is given by the potential drop across the Stern layer (bounded by the OHP) and the potential across the diffuse layer, the latter being given by the zeta potential. (c) Thickness of the Stern layer $\left[d_{\text {Stern }}\right.$, from Eq. (5)] as a function of hydrated cation radius, taking the reference potential $\Phi_{0}^{\mathrm{NaCl}}$ to be $-385 \pm 20 \mathrm{mV}$, as determined from Fig. 3 . The linear fit has a $y$ intercept at $1.4 \pm 0.6 \AA$.

[46]), providing evidence that $50-\mathrm{mM} \mathrm{Li}^{+}, \mathrm{Na}^{+}, \mathrm{K}^{+}$, and $\mathrm{Cs}^{+}$all adsorb via nonspecific electrostatic interactions at the water-silica NP interface. Moreover, a linear extrapolation of the experimental data to the infinitesimally small cation limit reveals a nonzero limiting Stern layer thickness $(1.4 \pm 0.6 \AA)$, consistent with a single hydration shell of water. A simple, intuitive and consistent interpretation of Fig. 5(c) is that the Stern layer around a silica NP consists of one molecular layer of water ( 2-3 $\AA$ ) [46], which hydrates the deprotonated silanol group and blocks cations from approaching the surface any further. This yields an OHP located one water layer plus one hydrated cation radius from the surface [Fig. 5(c)]. This single water layer is 
consistent with AFM measurements that show a tightly bound hydration layer on negatively charged silica surfaces [3]. Moreover, x-ray reflectivity measurements indicate no structuring beyond the first layer of adsorbed water molecules at a similar silica surface [47], and sum-frequency spectroscopy results show the structure of water at fused silica interfaces is independent of cation $\left(\mathrm{Li}^{+}, \mathrm{Na}^{+}, \mathrm{K}^{+}\right)$in 50-mM chloride electrolytes [48].

The capacitance of the Stern layer $\left(C_{\text {Stern }}=\varepsilon \varepsilon_{0} / d_{\text {Stern }}\right)$ follows naturally after $d_{\text {Stern }}$ has been determined. The capacitances increase as the size of the hydrated cation decreases, 0.48 in $\mathrm{LiCl}, 0.51$ in $\mathrm{NaCl}, 0.63$ in $\mathrm{KCl}$, and $0.83 \mathrm{~F} / \mathrm{m}^{2}$ in $\mathrm{CsCl}$ (Table II) — values that deviate considerably between cations because of their different distances from the NP surface, but that agree with those derived from regression of surface protonation data for amorphous silica [45].

\section{E. Modified Poisson-Boltzmann model with hydration repulsion}

During the past century, various mean-field modified Poisson-Boltzmann equations have been developed that incorporate an excess chemical potential that captures steric interactions between ions (see Refs. [49,50] and references therein). In particular, modified PB equations that employ the Carnahan-Starling expression for hardsphere gasses accurately reproduce the average ion densities that arise from molecular-dynamics computation of primitive model EDLs, yet fail to capture the Stern layer or any oscillatory structure near highly charged surfaces [51]. Strictly speaking, no mean-field model employing local-density approximations [e.g., lattice-ion (Bikerman) or hard sphere (CarnahanStarling)] can admit nonmonotonic EDL profiles [52].

Ions and surfaces in aqueous solutions often interact strongly with water molecules, and their hydration shells are known to mediate interactions [53,54]. At negatively charged surfaces like silica, cations reside in close proximity at concentrations that exceed bulk, whereas anions are depleted. Hence, one expects hydration interactions to be relevant only for cations because of their short interionic distances close to the surface. Moreover, such forces are responsible for the formation of the Stern layer. To capture such behavior, we employ a recent model [55-57] that adds a hydration repulsion between cations in the form of a Yukawa potential, $\left(U_{h} / k_{B} T\right)=b l_{h} \exp \left(\kappa l_{h}-\kappa r\right) / r$, to the Coulomb potential that is at the core of the classical PoissonBoltzmann model. Here, $k_{B}$ is the Boltzmann constant, $T$ the absolute temperature, $\kappa^{-1}=0.3 \mathrm{~nm}$ is the decay length of the water ordering [53], and $b$ is a constant that specifies the hydration interaction to equal $k_{B} T / b$ at a cation-cation distance $r=l_{h}$. Choosing $b=0.4$ allows the magnitude of $l_{h}$ to be identified with the reported sizes [37] of the hydrated cations (Table II). The motivation for choosing a

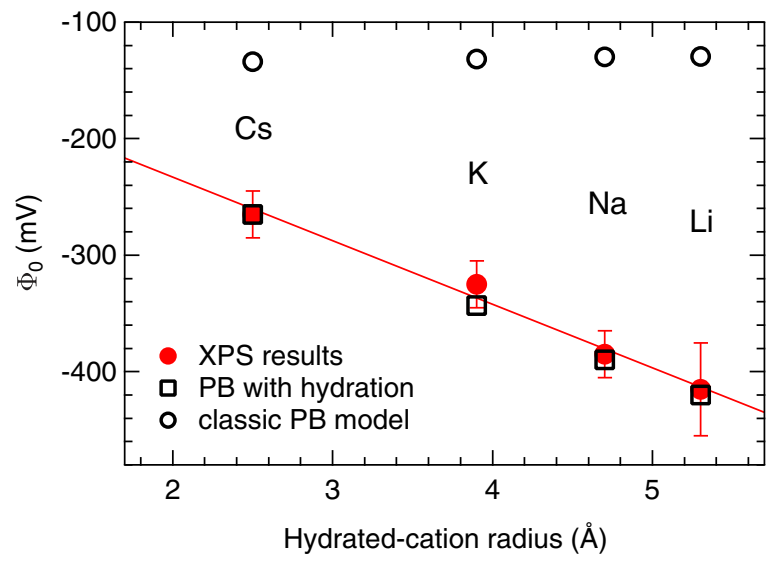

FIG. 6. Surface potentials at the silica NP-electrolyte interface. Red, solid circles: Results from XPS measurements. The solid line is a linear fit to the experimental data. Black, open squares: Prediction from the hydration-modified PB model. Black, open circles: Prediction from the Grahame equation based on the classic PB model.

Yukawa potential and the derivation of the hydrationmodified PB model are described in Appendix A 5.

We calculate the surface potentials of the silica-electrolyte interfaces with this hydration-modified PB model. Using the experimentally determined $\sigma_{\mathrm{NP}}^{\text {salt }}$ (Table I) together with the hydrated cation radii (Table II, see also Ref. [37]) yields surface potentials in quantitative agreement with experiments in all four electrolytes, within the reproducibility of the experiments (Fig. 6). This agreement can be rationalized by analyzing the concentration profiles, $n_{+}(x)$, of the four cations (solid lines in Fig. 7). Local maxima are predicted at finite separations from the interfaces that we interpret to be the formation of Stern-like layers [57], where

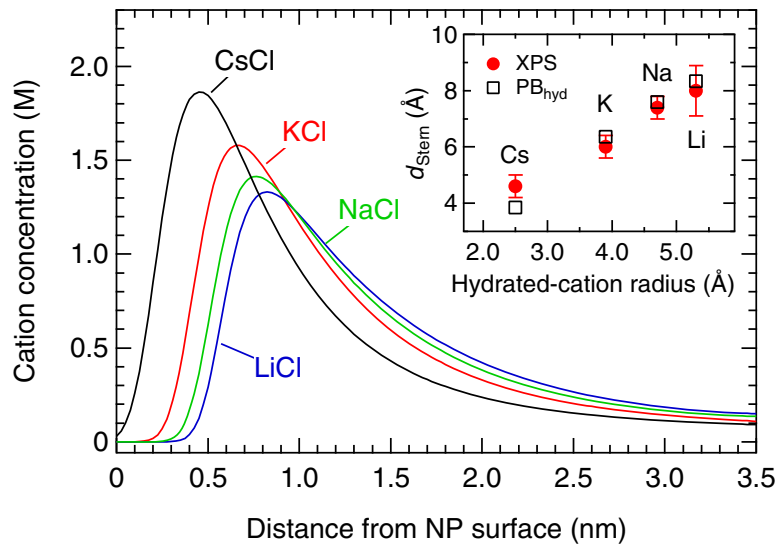

FIG. 7. Counterion concentration profiles in the electrical double layer. Cation concentration profiles as predicted by the hydration-modified PB model (solid lines) for 50-mM electrolyte: black, $\mathrm{CsCl}$; red, $\mathrm{KCl}$; green, $\mathrm{NaCl}$; blue $\mathrm{LiCl}$. Inset: Stern layer thicknesses calculated from experiments (red, closed symbols) and predicted by the hydration-modified PB model (black, open symbols). 
$d_{\text {Stern }}$ is chosen to be the maximum in $n_{+}(x)$ (inset of Fig. 7). Inside these Stern-like layers the potential drop is (nearly) linear (it behaves as a parallel-plate capacitor), giving rise to surface potentials that far exceed those predicted by the classical PB model (Grahame equation [58,59], open circles of Fig. 6). Ultimately, the hydrationmodified PB model, which retains much of the simplicity of the classical PB approach, successfully reproduces ion-specific surface potentials and Stern layer thicknesses at the silica-electrolyte interface as measured by XPS.

\section{DISCUSSION}

The operation of the liquid microjet for XPS experiments of dispersed NP suspensions is a challenging experiment and certain technical aspects of its operation might impact our measurements, and thus call our results into question. Here, we address the impact of streaming potential, the hydration state of the NPs, and x-ray beam damage.

Pressure-driven flow of an electrolyte solution through a liquid microjet naturally gives rise to so-called streaming potentials (or streaming currents) [60], which could, in principle, be significant in our experiments. Under essentially identical experimental conditions to ours (flow rate and capillary diameter), Kurahashi et al. showed streaming potentials of $-1.5 \mathrm{~V}$ in $5-\mathrm{mM}$ electrolyte $(\mathrm{NaCl}, \mathrm{NaBr}$, and $\mathrm{NaI})$ but concluded the addition of at least $30-\mathrm{mM}$ salt rendered the magnitude of this potential insignificant, allowing them to refine the $1 b_{1}$ orbital binding energy of liquid water to two decimal places (i.e., $\pm 0.04 \mathrm{eV}$ ) [22]. Lübcke and co-workers reached a similar conclusion in 2013 [61]. Our choice to add 50-mM electrolyte to the NP dispersions, besides providing a platform for the investigation of specific ion effects, is, therefore, in part chosen to ensure that streaming potentials associated with the operation of the liquid microjet are largely suppressed $[22,61]$. We include a more detailed discussion, including the calculation of the streaming potential, in Appendix A 6 . We anticipate streaming potentials of order $\sim 5-10 \mathrm{mV}$ at most in our experiments [can be compared with the smallest $\Delta \Phi_{0}$ between electrolytes in Fig. 2(c) $(\mathrm{LiCl}$ and $\mathrm{NaCl}$ ) of $30 \mathrm{mV}$ and our smallest error (derived from the standard deviation of repeat measurements) $\pm 20 \mathrm{mV}$ (Table II)]. Irrespective, however, any streaming potential that may persist affects both the $\mathrm{Si} 2 p$ and $\mathrm{O} 1 s$ BEs by precisely the same amount. Measured shifts in the Si $2 p \mathrm{BE}$ (emitted exclusively by the $\mathrm{NP}$ ) relative to the $\mathrm{O} 1 s \mathrm{BE}$ (emitted predominantly within the bulk electrolyte) truly reflects changes in the surface potential alone, irrespective of the streaming potential.

Although partial or altered hydration of the NPs probed in our measurements cannot be categorically excluded, we do not expect them to protrude from the aqueous solution into vacuum. Charge-stabilized colloidal silica NPs near air-water interfaces have been shown to remain completely hydrated, based on the interpretation of surface tension measurements using the Gibbs adsorption equation $[62,63]$, a finding that is consistent with the well-known hydrophilic nature of charge-stabilized colloidal silica [33]. We therefore expect the measured surface potentials to be likely representative of fully hydrated NPs in bulk solution conditions despite the limited probe depth of the XPS technique.

Any influence of $x$-ray beam damage on the results can be easily ruled out as the liquid jet is continuously refreshed at a rate of $7 \mathrm{~m} / \mathrm{s}$ [64]. Under these conditions the samples are exposed to $\mathrm{x}$ rays (spot size $0.1 \mathrm{~mm}$ ) [65] for a mere $\sim 20 \mu$ s before flowing past the beam. While this does not unambiguously rule out $\mathrm{x}$-ray beam damage, it would be difficult to anticipate $\mathrm{x}$-ray damage for $\mathrm{SiO}_{2}$ surface in $20 \mu$ s of x-ray exposure.

\section{CONCLUSIONS}

Combining XPS with a liquid microjet has allowed us to perform the very first measurement of the absolute surface potential of a NP suspended in an aqueous electrolyte. Based on this measurement, we use the Gouy-ChapmanStern model to compute the electrical double-layer structure at the water-silica $\left(\mathrm{SiO}_{2}\right) \mathrm{NP}$ interface, as influenced by specific cation effects. This simple approach reveals the Stern layer (bounded by the outer Helmholtz plane) to grow linearly with hydrated cation radius, and moreover to consist of a single layer of water molecules hydrating the silica surface, plus the hydrated radius of the cation, for all four cations $\left(\mathrm{Li}^{+}, \mathrm{Na}^{+}, \mathrm{K}^{+}\right.$, and $\left.\mathrm{Cs}^{+}\right)$. These results are broadly consistent with direct AFM imaging of ions within the Stern layer [4], and display an internal self-consistency that underscores its success. After all, our analysis naturally predicts a Stern layer thickness in the zero cation radius limit that qualitatively corresponds to a single layer of hydrating water molecules on an oxide surface. The magnitude of the surface potential increases $\left(\Phi_{0}\right.$ becomes more negative) with increasing hydrated cation size because they are held farther from the surface, yielding a larger potential drop across the Stern layer. We thus attribute the microscopic origins of the specific ion effects on the surface potential to the closer approach, and subsequent larger capacitance, of the Stern layer for smaller hydrated cations. A hydration-modified PoissonBoltzmann model that includes a soft nonelectrostatic interaction to describe the hydration repulsion between counterions quantitatively reproduces the measured specific ion effects using only the measured surface charge densities and the hydrated cation radii as input.

Finally, we note that the experimental determination of absolute surface potentials should work for a wide range of colloidal and NP suspensions in both aqueous and nonaqueous solvents. We therefore anticipate that these results are of interest to the electrochemistry community where an analogous strategy can be employed to tackle long-standing 
challenges related to quantifying energy-level alignment at liquid-semiconductor (quantum dot) interfaces [66-68].

\section{ACKNOWLEDGEMENTS}

A portion of this work was performed at the SIM beam line of the Swiss Light Source. M. A. B. and A. G. acknowledge funding from the Swiss National Science Foundation under Grant No. 153578, M. A. B. and S. M. the Swiss National Science Foundation International Short Visit program under Grant No. 162320, Z. A. the Nanosphere centre of excellence and Formas under Grant No. 2016-2013-673, and T.M.S. the U.S. National Science Foundation under Grant No. CBET-1438779 and ETH Zürich for support. A. Rossi, T. Vander Glas, and G. Granger are acknowledged for their help in the laboratory.

\section{APPENDIX: EXPERIMENTAL AND THEORETICAL METHODS}

\section{Materials}

All colloid experiments are carried out using Ludox SM silica (W.R. Grace and Company, Sigma-Aldrich). This colloidal suspension is sold as $30 \mathrm{wt} \%$ silica in $p \mathrm{H} 10$ aqueous solution. The nanoparticles are charge stabilized (surfactant free). Lithium chloride ( $\mathrm{LiCl}, \geq 99 \%$, ACS reagent, Sigma-Aldrich), sodium chloride $(\mathrm{NaCl}, \geq 99.8 \%$, ACS reagent, Sigma-Aldrich), potassium chloride $(\mathrm{KCl}$, 99.0\%-100.5\%, ACS reagent, Sigma-Aldrich), cesium chloride $\left(\mathrm{CsCl}, 99^{+} \%\right.$, Acros Organics), hydrochloric acid $(\mathrm{HCl}$, $0.1 \mathrm{~N}$, Acros Organics), and $\mathrm{HCl}$ (fuming ACS reagent, $\geq 37 \%$, Sigma-Aldrich) are used as received.

The stock Ludox SM suspension (30 wt $\% \mathrm{SiO}_{2}$ ) is dripped through $0.007-\mathrm{mm}$ pore-size filter paper (model 52 , Whatman). $5 \mathrm{wt} \%$ suspensions of the filtered sample are prepared in $50-\mathrm{mM}$ alkali chloride electrolytes using Milli-Q water. The suspensions are sonicated at room temperature for 5 min immediately after preparation and again for $5 \mathrm{~min}$ prior to their measurement. The four silica suspensions have $p \mathrm{H} 10.0 \pm 0.1$ as determined by a fourpoint $(2.00,4.01,7.00,10.00$, Technical Buffer Solutions, Mettler-Toledo) calibrated Mettler-Toledo ExpertPro electrode. $\mathrm{HCl}$ is used to acidify the suspensions for $p \mathrm{H}$-dependent measurements.

\section{Potentiometric titrations}

Surface charge densities are determined by potentiometric titration using a Mettler-Toledo G20 Compact Titrator equipped with a Mettler-Toledo Expert electrode and an electronic controlled rod stirrer $(70 \%$ polypropylene and $30 \%$ fiberglass). Polypropylene sample beakers of $100 \mathrm{~mL}$ are used. Experiments are performed for 5 wt $\%$ silica (Ludox SM) in $50-\mathrm{mM} \mathrm{LiCl}, \mathrm{NaCl}, \mathrm{KCl}$, or $\mathrm{CsCl}$ at $295 \mathrm{~K}$. The electrode is calibrated using a four-point curve (2.00, 4.01, 7.00, 10.00, Technical Buffer Solutions, MettlerToledo) immediately prior to every experiment. Suspensions of $25-\mathrm{mL}$ volume are titrated from high $p \mathrm{H}$ to low using $50-\mathrm{mM}$ electrolyte ( $\mathrm{LiCl}, \mathrm{NaCl}, \mathrm{KCl}$, and $\mathrm{CsCl}$ ) in $0.1-\mathrm{M}$ $\mathrm{HCl}$ (Acros Organics). The addition of 50-mM electrolyte to the $\mathrm{HCl}$ solution ensures that the concentration of electrolyte remains at $50 \mathrm{mM}$ throughout the entire titration. Experiments are performed in an inert atmosphere of nitrogen $\left(\mathrm{N}_{2}\right)$ gas that is bubbled through Milli-Q water. The drop volume of the $\mathrm{HCl}$ is set as $0.2 \mathrm{~mL}$ per step for the silica sample and $0.005 \mathrm{~mL}$ per step for the blank (50-mM electrolyte, no $\mathrm{SiO}_{2}$ ). The stir rate (electronic stir bar) of the suspension and blank samples are $700 \mathrm{rpm}$. The end point is set at $p \mathrm{H} 3.0$ [69]. SCDs are calculated following the procedure described by Lützenkirchen et al. [70] with a specific surface area of $282 \mathrm{~m}^{2} / \mathrm{g}$. Measurements are performed in triplicate to ensure reproducibility.

\section{Laboratory-based x-ray photoelectron spectroscopy}

A boron-doped (100)-oriented Si single crystal wafer (MTI Corporation) is used. XPS measurements are performed using a Kratos Axis Ultra DLD spectrometer system and a monochromatic $\mathrm{Al} \mathrm{K} \alpha(1486.7 \mathrm{eV})$ x-ray source. The sample bias potential is provided by a Keithley 2410 de power supply through the sample holder and its copper contacts clamped to the sample surface. The power supply is calibrated against an HP 3458 voltmeter, which itself is directly traceable to the National Research Council Canada Josephson array. Spectra of the $\mathrm{Si} 2 p$ region are acquired at $0.025 \mathrm{eV}$ step size, $2 \mathrm{~s}$ dwell, and $10 \mathrm{eV}$ pass energy. A single spectrum is taken at multiple nonzero sample bias voltages $( \pm 0.1, \pm 0.2, \pm 0.5, \pm 1.0, \pm 2.0$, and $\pm 5.0 \mathrm{~V}$ ). Following each bias a spectrum is collected at $0 \mathrm{~V}$ (we refer to this point as grounded). In this way any dynamic binding energy changes due to sample charging could be corrected. At most, this procedure results in a correction of $0.01 \mathrm{eV}$, but in most cases no correction is needed. The combined uncertainty in the experimental slope is dominated by the XPS spectrum step size and repeatability (including data processing) and is estimated to be less than $0.01 \mathrm{~V} / \mathrm{eV}$ (1 standard deviation). The uncertainty contribution due to calibration of the power supply and spectrometer linearity is negligible.

\section{Liquid microjet $x$-ray photoelectron spectroscopy}

XPS experiments are performed at the SIM beam line [26] of the Swiss Light Source using a 0.032-mm liquid microjet operating at $279 \mathrm{~K}$ and a flow rate of $0.45 \mathrm{~mL} / \mathrm{min}$ to continuously drive silica NPs in aqueous suspension through the beam, while maintaining an evacuated environment for the photoelectrons to traverse and be collected. A complete description of in situ XPS at the three-way interface of air-water-colloid is given elsewhere [71]. The Swiss Light Source near ambient pressure photoemission end station is used [20]. All experiments are performed in vacuum $\left(1 \times 10^{-4} \mathrm{mbar}\right)$. The liquid jet is expanded to hit a $\mathrm{LN}_{2}$ trap (so that it is frozen immediately 
and effectively removed), and the measurement chamber is continuously pumped by an Agilent TwisTorr 700 turbo molecular pump backed by an Adixen Roots pump. The Scienta R4000 HiPP-2 spectrometer is operated in constant energy mode at a pass energy of $50 \mathrm{eV}$. The entrance slit to the hemisphere is 0.3 (width) $\times 25$ (length) $\mathrm{mm}$ (curved), which results in an energy resolution better than $40 \mathrm{meV}$. The liquid jet is operated along the vertical length of the entrance slit. The entrance cone of the hemispherical energy analyzer (NAPP) has an aperture of $0.5 \mathrm{~mm}$ with a working distance to the surface of the liquid microjet of $0.5 \mathrm{~mm}$.

The primary photon energy [with linear polarization (>97\% [26]) along the spectrometer lens axis and perpendicular to the direction of liquid propagation] is set to $420 \mathrm{eV}$ to ionize the Si $2 p$ orbital, and second-order light [72], $840 \mathrm{eV}$, with $10 \%$ the intensity of the primary beam simultaneously ionized the O $1 s$ orbital. This allows $\mathrm{O} 1 s$ and $\mathrm{Si} 2 p$ photoelectrons to be collected within $10-\mathrm{eV}$ kinetic energy of each other, ensuring a constant probe depth to the experiment. There is no peak in the $\mathrm{Si} 2 p$ region of the spectrum when the NPs are not present (Fig. 8). Moreover, because we reference the Si $2 p$ BEs to that of $\mathrm{O} 1 s$ from liquid water-assumed constant between the different NP suspensions-this approach ensures that any residual streaming potential that may persist in $50-\mathrm{mM}$ electrolyte is corrected: A streaming potential would affect the kinetic energy of both the $\mathrm{Si} 2 p$ and the $\mathrm{O} 1 s$ photoelectrons in exactly the same manner. Our assumption that the $\mathrm{O} 1 s \mathrm{BE}$ is constant between solutions is likely valid in $50-\mathrm{mM}$ electrolyte where there are 539 water molecules for each ion in solution (for $\mathrm{NaCl}$ ) [73]. With the conservative estimate that the solvation shell of each ion requires 10 water molecules, only $2 \%$ of all water is directly involved in solvation. No shift in $\mathrm{O} 1 s \mathrm{BE}$ is expected between the different electrolyte solutions because the signal is dominated by the $98 \%$ of water molecules that

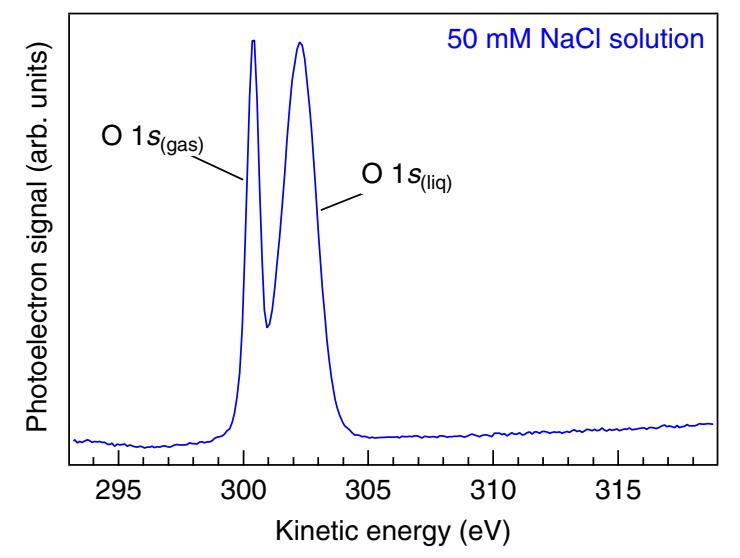

FIG. 8. Reference $\mathrm{O} 1 \mathrm{~s}$ spectrum in the absence of nanoparticles. Spectrum of $50-\mathrm{mM} \mathrm{NaCl}$ solution (no silica NPs) under identical conditions to those presented in Fig. 2(b). The region between 310 and $314 \mathrm{eV}$ is flat in the absence of the nanoparticles. are indifferent to the presence of the electrolyte. Specific ion effect measurements at $p \mathrm{H} 10$ [Fig. 2(b)] are repeated in duplicate, and over two different beam times (four measurements in total). $p \mathrm{H}$-dependent measurements in $\mathrm{NaCl}$ (Fig. 3) are repeated in duplicate during a single beam time.

\section{Modified Poisson-Boltzmann model with hydration repulsion}

Our choice of a Yukawa (screened Coulomb) interaction to describe the nonelectrostatic repulsion between cations and between cations and the surface is motivated by the hydration forces model of Marcelja and Radic [74]. Computer simulations predict a similar decay [75], albeit with an additional oscillatory component that is omitted here for simplicity. The total cation-cation interaction energy in this hydrationmodified PB model is given by $\left(U_{\text {tot }} / k_{B} T\right)=$ $\left(U_{e} / k_{B} T\right)+\left(U_{h} / k_{B} T\right)=l_{e} / r+b l_{h} \exp \left(\kappa l_{h}-\kappa r\right) / r$, where $l_{e}=0.7 \mathrm{~nm}$ is the Bjerrum length. The cation-anion and anion-anion interactions remain purely electrostatic.

Analogous to translating the Coulomb interaction, $\left(U_{e} / k_{B} T\right)=l_{e} / r$, to the Poisson equation,

$$
\Psi_{e}^{\prime \prime}(x)=-4 \pi l_{e}\left[n_{+}(x)-n_{-}(x)\right],
$$

for an associated dimensionless electrostatic potential $\Psi_{e}$, with the local cation $\left(n_{+}(x)\right)$ and anion $\left(n_{-}(x)\right)$ concentrations, the hydration interaction, $\left(U_{h} / k_{B} T\right)=$ $b l_{h} \exp \left(\kappa l_{h}-\kappa r\right) / r$, can be translated to a local differential equation for an associated dimensionless hydration potential $\Psi_{h}$. This results in the inhomogeneous Helmholtz equation [56],

$$
\Psi_{h}^{\prime \prime}(x)-\kappa^{2} \Psi_{h}(x)=-4 \pi b l_{h} \exp \left(\kappa l_{h}\right)\left[n_{+}(x)-n_{0}\right],
$$

where $n_{0}$ is the bulk ion concentration $(50 \mathrm{mM}$ in the experiments). The local cation and anion concentrations fulfill Boltzmann distributions:

$$
\begin{aligned}
& n_{-}(x)=n_{0} \exp \left[\Psi_{e}(x)\right], \\
& n_{+}(x)=n_{0} \exp \left[-\Psi_{e}(x)-\Psi_{h}(x)\right] .
\end{aligned}
$$

These Boltzmann distributions are derived rigorously by minimizing an appropriate mean-field free-energy functional [56]. The dimensionless hydration potential $\Psi_{h}$ is associated only with the cations because the local anion concentration remains dilute throughout the entire aqueous solution. Inserting these Boltzmann distributions into Eqs. (A1) and (A2) yields two coupled differential equations for the two fields $\Psi_{e}(x)$ and $\Psi_{h}(x)$,

$\Psi_{e}^{\prime \prime}(x)=\frac{\kappa_{e}^{2}}{2}\left\{\exp \left[\Psi_{e}(x)\right]-\exp \left[-\Psi_{e}(x)-\Psi_{h}(x)\right]\right\}$, 
$\Psi_{h}^{\prime \prime}(x)-\kappa^{2} \Psi_{h}(x)=\frac{\kappa_{h}^{2}}{2}\left\{1-\exp \left[-\Psi_{e}(x)-\Psi_{h}(x)\right]\right\}$,

where $\kappa_{e}^{2}=8 \pi l_{e} n_{0}, \kappa_{e}^{-1}$ is the Debye length and $\kappa_{h}^{2}=$ $8 \pi b l_{h} \exp \left(\kappa l_{h}\right) n_{0}$. For a single planar charged surface, four boundary conditions need to be specified. Two of them, $\Psi_{e}^{\prime}(x=0)=-4 \pi l_{e} \sigma_{\mathrm{NP}}$ and $\Psi_{h}^{\prime}(x=0)=$ $-4 \pi b \exp \left(\kappa l_{h}\right) l_{h} \sigma_{h}$, reflect the surface charge density $\sigma_{\mathrm{NP}}$ and the density of sources for the hydration interaction of the cations with the surface $\sigma_{h}$, respectively. A reasonable choice for the latter is the surface density of water molecules, $\sigma_{h}=5 \mathrm{~nm}^{-2}$. The remaining boundary conditions, $\Psi_{e}^{\prime}(x \rightarrow \infty)=0$ and $\Psi_{h}^{\prime}(x \rightarrow \infty)=0$, render the surface isolated.

\section{Streaming potential}

A streaming potential can occur across the liquid microjet nozzle during its operation. It is caused by an asymmetric charge displacement in the electrical double layer of the capillary as a result of an applied pressure inducing the liquid NP suspension to move tangentially to the wall of the capillary. Streaming potentials are calculated from [60]

$$
\Phi_{\text {streaming }}=\frac{\varepsilon \varepsilon_{0} \zeta}{\eta K} \Delta P,
$$

where $\zeta$ is the (unknown) zeta potential of the inner walls of the $0.032-\mathrm{mm}$ (diameter) quartz capillary, $\eta$ and $K$ are the viscosity and conductivity of the NP suspension, respectively, and $\Delta P$ the pressure used to drive liquid flow (2 bar). Here, we use the experimentally determined zeta potentials for colloidal silica (Table II, see also Ref. [35]), while treating the viscosity between the four different NP suspensions as constant. The latter is justified by the (relatively high concentration) $5 \mathrm{wt} \% \mathrm{SiO}_{2}$ samples we investigate- the viscosity of the suspensions (5.5 MPa s for the stock $30 \mathrm{wt} \%$ sample) is dominated by the NP concentration and not by the electrolyte. The conductivity of aqueous solutions of 50-mM $\mathrm{NaCl}$ and $\mathrm{KCl}$ are identical $\left(8.2 \mathrm{mS} \mathrm{cm}^{-1}\right)$ while that of $\mathrm{LiCl}$ is $10.1 \mathrm{mS} \mathrm{cm}^{-1}$ [73]. Using the dielectric constant of bulk water (78), and the viscosity of pure water as a lower bound $\left(1.3 \times 10^{-3} \mathrm{Pas}\right)$ [73] (which gives an upper bound to the streaming potential), we calculate a streaming potential of $6 \pm 5 \mathrm{mV}$ for all suspensions. The standard deviation of the XPS measurements (Fig. 2 and Table II) is reported as a minimum of $\pm 20 \mathrm{mV}$-a factor of 3 greater than the predicted streaming potentials. The similarity between the different solutions and the negligible magnitude of the streaming potential is consistent with the independent experimental reports of Suzuki and co-workers [22] and Lübcke and co-workers [61].

Furthermore, if a small residual streaming potential were to persist in these suspensions it would be corrected for in the data analysis, and would therefore be irrelevant to the conclusions of this article. In this case, the streaming potential would affect equally the $\mathrm{Si} 2 p$ and $\mathrm{O} 1 s$
BEs-a common mode shift. The latter is presumed constant between the different suspensions and is the only suitable reference state for aqueous solution-based XPS.

[1] T. J. Su, J. R. Lu, R. K. Thomas, and J. Penfold, Neutron Reflection from Counterions at the Surface of a Soluble Surfactant Solution, J. Phys. Chem. B 101, 937 (1997).

[2] T. J. Su, R. K. Thomas, and J. Penfold, Neutron Reflection from Counterions at the Surface Formed by a Charged Insoluble Monolayer, Langmuir 13, 2133 (1997).

[3] J. Morag, M. Dishon, and U. Sivan, The Governing Role of Surface Hydration in Ion Specific Adsorption to Silica: An AFM-Based Account of the Hofmeister Universality and Its Reversal, Langmuir 29, 6317 (2013).

[4] I. Siretanu, D. Ebeling, M. P. Andersson, S. L. S. Stipp, A. Philipse, M. C. Stuart, D. van den Ende, and F. Mugele, Direct Observation of Ionic Structure at Solid-Liquid Interfaces: A Deep Look Into the Stern Layer, Sci. Rep. 4, 4956 (2014).

[5] L. Wang, C. L. Zhao, M. H. G. Duits, F. Mugele, and I. Siretanu, Detection of Ion Adsorption at Solid-Liquid Interfaces Using Internal Reflection Ellipsometry, Sens. Actuators B 210, 649 (2015).

[6] M. F. Toney, J. N. Howard, J. Richer, G. L. Borges, J. G. Gordon, O. R. Melroy, D. G. Wiesler, D. Yee, and L. B. Sorensen, Voltage-Dependent Ordering of Water-Molecules at an Electrode-Electrolyte Interface, Nature (London) $\mathbf{3 6 8}$, 444 (1994).

[7] S. Nihonyanagi, S. Yamaguchi, and T. Tahara, Water Hydrogen Bond Structure near Highly Charged Interfaces Is Not Like Ice, J. Am. Chem. Soc. 132, 6867 (2010).

[8] C. A. Bunton, F. Nome, F. H. Quina, and L. S. Romsted, Ion Binding and Reactivity at Charged Aqueous Interfaces, Acc. Chem. Res. 24, 357 (1991).

[9] W. B. Russel, D. A. Saville, and W. R. Schowalter, Colloidal Dispersions (Cambridge University Press, Cambridge, England, 1999).

[10] T. M. Squires and S. R. Quake, Microfluidics: Fluid Physics at the Nanoliter Scale, Rev. Mod. Phys. 77, 977 (2005).

[11] W. Sparreboom, A. van den Berg, and J.C. T. Eijkel, Principles and Applications of Nanofluidic Transport, Nat. Nanotechnol. 4, 713 (2009).

[12] Y. Jiang, R. Tang, B. Duncan, Z. Jiang, B. Yan, R. Mout, and V. M. Rotello, Direct Cytosolic Delivery of siRNA Using Nanoparticle-Stabilized Nanocapsules, Angew. Chem., Int. Ed. Engl. 54, 506 (2015).

[13] B. E. Conway, Electrochemical Supercapacitors: Scientific Fundamentals and Technological Applications (Plenum Publishers, New York, 1999).

[14] S. Porada, B. B. Sales, H. V. M. Hamelers, and P. M. Biesheuvel, Water Desalination with Wires, J. Phys. Chem. Lett. 3, 1613 (2012).

[15] S. Porada, L. Weinstein, R. Dash, A. van der Wal, M. Bryjak, Y. Gogotsi, and P. M. Biesheuvel, Water Desalination Using Capacitive Deionization with Microporous Carbon Electrodes, ACS Appl. Mater. Interfaces 4, 1194 (2012). 
[16] N. S. Choi, Z. Chen, S. A. Freunberger, X. Ji, Y.-K. Sun, K. Amine, G. Yushin, L. F. Nazar, J. Cho, and P. G. Bruce, Challenges Facing Lithium Batteries and Electrical Double-Layer Capacitors, Angew. Chem., Int. Ed. Engl. 51, 9994 (2012).

[17] J. Lyklema, Fundamentals of Interface and Colloid Science (Academic Press, San Diego, 1995), Vol. II.

[18] D. A. Sverjensky, Interpretation and Prediction of Triple-Layer Model Capacitances and the Structure of the Oxide-Electrolyte-Water Interface, Geochim. Cosmochim. Acta 65, 3643 (2001).

[19] J. Lutzenkirchen, T. Preocanin, F. Stipic, F. Heberling, J. Rosenqvist, and N. Kallay, Surface Potential at the Hematite (001) Crystal Plane in Aqueous Environments and the Effects of Prolonged Aging in Water, Geochim. Cosmochim. Acta 120, 479 (2013).

[20] M. A. Brown et al., A New Endstation at the Swiss Light Source for Ultraviolet Photoelectron Spectroscopy, X-Ray Photoelectron Spectroscopy, and X-Ray Absorption Spectroscopy Measurements of Liquid Solutions, Rev. Sci. Instrum. 84, 073904 (2013).

[21] M. A. Brown, M. Faubel, and B. Winter, X-Ray Photo- and Resonant Auger-Electron Spectroscopy Studies of Liquid Water and Aqueous Solutions., Annual reports on the progress of chemistry Section A, Inorganic chemistry 105, 174 (2009).

[22] N. Kurahashi, S. Karashima, Y. Tang, T. Horio, B. Abulimiti, Y. I. Suzuki, Y. Ogi, M. Oura, and T. Suzuki, Photoelectron Spectroscopy of Aqueous Solutions: Streaming Potentials of $\mathrm{NaX}(\mathrm{X}=\mathrm{Cl}, \mathrm{Br}$, and I) Solutions and Electron Binding Energies of Liquid Water and $\mathrm{X}^{-}$, J. Chem. Phys. 140, 174506 (2014).

[23] H. Bluhm et al., Soft X-Ray Microscopy and Spectroscopy at the Molecular Environmental Science Beamline at the Advanced Light Source, J. Electron Spectrosc. Relat. Phenom. 150, 86 (2006).

[24] M. M. Walz, C. Caleman, J. Werner, V. Ekholm, D. Lundberg, N. L. Prisle, G. Ohrwall, and O. Bjorneholm, Surface Behavior of Amphiphiles in Aqueous Solution: A Comparison Between Different Pentanol Isomers, Phys. Chem. Chem. Phys. 17, 14036 (2015).

[25] M. A. Brown, A. Beloqui Redondo, M. Sterrer, B. Winter, G. Pacchioni, Z. Abbas, and J. A. van Bokhoven, Measure of Surface Potential at the Aqueous-Oxide Nanoparticle Interface by XPS from a Liquid Microjet., Nano Lett. 13, 5403 (2013).

[26] U. Flechsig, F. Nolting, A. F. Rodriguez, J. Krempasky, C. Quitmann, T. Schmidt, S. Spielmann, and D. Zimoch, Performance Measurements at the SLS SIM Beamline, AIP Conf. Proc. 1234, 319 (2010).

[27] M. A. Brown, M. Arrigoni, F. Heroguel, A. Beloqui Redondo, L. Giordano, J. A. van Bokhoven, and G. Pacchioni, pH Dependent Electronic and Geometric Structures at the Water-Silica Nanoparticle Interface, J. Phys. Chem. C 118, 29007 (2014).

[28] B. V. Crist, Handbook of Monochromatic XPS Spectra, Semiconductors (Wiley, New York, 2000).

[29] B. V. Crist, Handbook of Monochromatic XPS Spectra, The Elements of Native Oxides (Wiley, New York, 2000).
[30] S. Suzer and A. Dana, X-Ray Photoemission for Probing Charging/Discharging Dynamics., J. Phys. Chem. B 110, 19112 (2006).

[31] B. Ulgut and S. Suzer, XPS Studies of $\mathrm{SiO}_{2} / \mathrm{Si}$ System Under External Bias, J. Phys. Chem. B 107, 2939 (2003).

[32] E. J. Crumlin, H. Bluhm, and L. Zhi, In Situ Investigations of Electrochemical Devices Using Ambient Pressure Photoelectron Spectroscopy, J. Electron Spectrosc. Relat. Phenom. 190, 84 (2013).

[33] R. K. Iler, The Chemistry of Silica: Solubility, Polymerization, Colloid and Surface Properties and Biochemistry of Silica (Wiley, New York, 1979).

[34] G. A. Parks, The Isoelectric Points of Solid Oxides Solid Hydroxides and Aqueous Hydroxo Complex Systems, Chem. Rev. 65, 177 (1965).

[35] A. Beloqui Redondo, I. Jordan, I. Ziazadeh, A. Kleibert, J. B. Giorgi, H. J. Wörner, S. May, Z. Abbas, and M. A. Brown, Nanoparticle-Induced Charge Redistribution of the Air-Water Interface, J. Phys. Chem. C 119, 2661 (2015).

[36] G. V. Franks, Zeta Potentials and Yield Stresses of Silica Suspensions in Concentrated Monovalent Electrolytes: Isoelectric Point Shift and Additional Attraction, J. Colloid Interface Sci. 249, 44 (2002).

[37] J. Kielland, Individual Activity Coefficients of Ions in Aqueous Solutions, J. Am. Chem. Soc. 59, 1675 (1937).

[38] S. W. Ong, X. L. Zhao, and K. B. Eisenthal, Polarization of Water-Molecules at a Charged Interface-2nd Harmonic Studies of the Silica Water Interface, Chem. Phys. Lett. 191, 327 (1992).

[39] L. Bousse, N. F. Derooij, and P. Bergveld, Operation of Chemically Sensitive Field-Effect Sensors as a Function of the Insulator-Electrolyte Interface, IEEE Trans. Electron Devices 30, 1263 (1983).

[40] W. M. Siu and R. S. C. Cobbold, Basic Properties of the Electrolyte-SiO2-Si System-Physical and Theoretical Aspects, IEEE Trans. Electron Devices 26, 1805 (1979).

[41] J. L. Diot, J. Joseph, J. R. Martin, and P. Clechet, $p H$ Dependence of the $\mathrm{Si} / \mathrm{SiO}_{2}$ Interface State Density for EOS Systems, J. Electroanal. Chem. 193, 75 (1985).

[42] L. Bousse and J. D. Meindl, in Geochemical Processes at Mineral Surfaces, ACS Symposium Series Vol. 323, edited by J. A. Davis and K. F. Hayes (American Chemical Society, Washington, DC, 1986), Chap. 5, pp. 79-98.

[43] R. R. Kumal, T. E. Karam, and L. H. Haber, Determination of the Surface Charge Density of Colloidal Gold Nanoparticles Using Second Harmonic Generation, J. Phys. Chem. C 119, 16200 (2015).

[44] D. J. Shaw, Introduction to Colloid and Surface Chemistry, 4th ed. (Butterworth-Heinemann Ltd., Oxford, 1992).

[45] D. A. Sverjensky, Prediction of Surface Charge on Oxides in Salt Solutions: Revisions for 1:1 $\left(\mathrm{M}^{+} \mathrm{L}^{-}\right)$Electrolytes, Geochim. Cosmochim. Acta 69, 225 (2005).

[46] A. Poynor, L. Hong, I. K. Robinson, S. Granick, Z. Zhang, and P. A. Fenter, How Water Meets a Hydrophobic Surface, Phys. Rev. Lett. 97, 266101 (2006).

[47] M. L. Schlegel, K. L. Nagy, P. Fenter, and N. C. Sturchio, Structures of Quartz (100)- and (101)-Water Interfaces Determined by X-Ray Reflectivity and Atomic Force Microscopy of Natural Growth Surfaces, Geochim. Cosmochim. Acta 66, 3037 (2002). 
[48] Z. Yang, Q. F. Li, and K. C. Chou, Structures of Water Molecules at the Interfaces of Aqueous Salt Solutions and Silica: Cation Effects, J. Phys. Chem. C 113, 8201 (2009).

[49] M. Z. Bazant, M. S. Kilic, B. D. Storey, and A. Ajdari, Towards an Understanding of Induced-Charge Electrokinetics at Large Applied Voltages in Concentrated Solutions, Adv. Colloid Interface Sci. 152, 48 (2009).

[50] A. A. Kornyshev, Double-Layer in Ionic Liquids: Paradigm Change?, J. Phys. Chem. B 111, 5545 (2007).

[51] B. Giera, N. Henson, E. M. Kober, M. S. Shell, and T. M. Squires, Electric Double-Layer Structure in Primitive Model Electrolytes: Comparing Molecular Dynamics with Local-Density Approximations, Langmuir 31, 3553 (2015).

[52] B. Giera, N. Henson, E. M. Kober, T. M. Squires, and M. S. Shell, Model-Free Test of Local-Density Mean-Field Behavior in Electric Double Layers, Phys. Rev. E 88, 011301(R) (2013).

[53] J. N. Israelachvili and P. M. Mcguiggan, Forces between Surfaces in Liquids, Science 241, 795 (1988).

[54] K. A. Dill, T. M. Truskett, V. Vlachy, and B. Hribar-Lee, Modeling Water, the Hydrophobic Effect, and Ion Solvation, Annu. Rev. Biophys. Biomol. Struct. 34, 173 (2005).

[55] K. Bohinc, A. Shrestha, and S. May, The Poisson-HelmholtzBoltzmann Model, Eur. Phys. J. E 34, 108 (2011).

[56] K. Bohinc, A. Shrestha, M. Brumen, and S. May, PoissonHelmholtz-Boltzmann Model of the Electric Double Layer: Analysis of Monovalent Ionic Mixtures, Phys. Rev. E 85, 031130 (2012).

[57] M. A. Brown, G. Volpe Bossa, and S. May, Emergence of a Stern Layer from the Incorporation of Hydration Interactions into the Gouy-Chapman Model of the Electrical Double Layer, Langmuir 31, 11477 (2015).

[58] H.-J. Butt, K. Graf, and M. Kappl, Physics and Chemistry of Interfaces, 3rd ed. (Wiley-VCH Verlag $\mathrm{GmbH}$, Weinheim, 2013).

[59] D. C. Grahame, The Electrical Double Layer and the Theory of Electrocapillarity, Chem. Rev. 41, 441 (1947).

[60] A. V. Delgado, E. Gonzalez-Caballero, R. J. Hunter, L. K. Koopal, and J. Lyklema, Measurement and Interpretation of Electrokinetic Phenomena (IUPAC Technical Report), Pure Appl. Chem. 77, 1753 (2005).

[61] N. Preissler, F. Buchner, T. Schultz, and A. Lübcke, Electrokinetic Charging and Evidence for Charge Evaporation in Liquid Microjets of Aqueous Salt Solution, J. Phys. Chem. B 117, 2422 (2013).

[62] I. Blute, R. J. Pugh, J. van de Pas, and I. Callaghan, Industrial Manufactured Silica Nanoparticle Sols. 2: Surface Tension, Particle Concentration, Foam Generation and Stability, Colloid Surf. A 337, 127 (2009).

[63] M. A. Brown, N. Duyckaerts, A. Beloqui Redondo, I. Jordan, F. Nolting, A. Kleibert, M. Ammann, H. Jakob Wörner, J.A. van Bokhoven, and Z. Abbas, Effect of
Surface Charge Density on the Affinity of Oxide Nanoparticles for the Vapor-Water Interface, Langmuir 29, 5023 (2013).

[64] M. Faubel, B. Steiner, and J. P. Toennies, Photoelectron Spectroscopy of Liquid Water, Some Alcohols, and Pure Nonane in Free Micro Jets, J. Chem. Phys. 106, 9013 (1997).

[65] G. Olivieri, A. Goel, A. Kleibert, and M. A. Brown, Effect of X-Ray Spot Size on Liquid Jet Photoelectron Spectroscopy, J. Synchrotron Radiat. 22, 1528 (2015).

[66] A. J. Bard, A. B. Bocarsly, F. R. F. Fan, E. G. Walton, and M. S. Wrighton, The Concept of Fermi Level Pinning at Semiconductor-Liquid Junctions-Consequences for Energy-Conversion Efficiency and Selection of Useful Solution Redox Couples in Solar Devices, J. Am. Chem. Soc. 102, 3671 (1980).

[67] J. H. Bang and P. V. Kamat, Quantum Dot Sensitized Solar Cells. A Tale of Two Semiconductor Nanocrystals: CdSe and CdTe, ACS Nano 3, 1467 (2009).

[68] N. Kharche, J. T. Muckerman, and M. S. Hybertsen, FirstPrinciples Approach to Calculating Energy Level Alignment at Aqueous Semiconductor Interfaces, Phys. Rev. Lett. 113, 176802 (2014).

[69] S. K. Milonjic, Determination of Surface-Ionization and Complexation Constants at Colloidal Silica Electrolyte Interface, Colloid Surf. 23, 301 (1987).

[70] J. Lützenkirchen, T. Preocanin, D. Kovacevic, V. Tomisic, L. Lovgren, and N. Kallay, Potentiometric Titrations as a Tool for Surface Charge Determination, Croat. Chem. Acta $\mathbf{8 5}$, 391 (2012).

[71] M. A. Brown, I. Jordan, A. Beloqui Redondo, A. Kleibert, H. J. Wörner, and J. A. van Bokhoven, In Situ Photoelectron Spectroscopy at the Liquid/Nanoparticle Interface, Surf. Sci. 610, 1 (2013).

[72] Second order also light fulfills the grating equation, $n \lambda=d[\sin (i)+\sin (r)]$, at the beam line monochromator, where $n$ is a positive integer, $\lambda$ is the wavelength of $\mathrm{x}$-ray light, $d$ is the spacing between the reflecting surfaces, and $i$ and $r$ are the incident and reflected angles, respectively. For the primary photon energy $(420 \mathrm{eV}), n=1$, whereas $n=2$ for second-order light $(840 \mathrm{eV})$. The restriction in the grating equation that $n$ be an integer for the interference to be constructive ensures that the second-order $\mathrm{x}$ ray has exactly half the wavelength, and therefore exactly double the energy, of the primary $\mathrm{x}$ ray.

[73] CRC Handbook of Chemistry and Physics, 56th ed. (CRC Press, Cleveland, OH, 1975).

[74] S. Marcelja and N. Radic, Repulsion of Interfaces due to Boundary Water, Chem. Phys. Lett. 42, 129 (1976).

[75] I. Kalcher, J. C. F. Schulz, and J. Dzubiella, Ion-Specific Excluded-Volume Correlations and Solvation Forces, Phys. Rev. Lett. 104, 097802 (2010). 